\title{
Acoustic noise in deep ice and environmental conditions at the South Pole
}

\author{
Timo Karg for the IceCube collaboration \\ Bergische Universität Wuppertal, Fachbereich C - Mathematik und Naturwissenschaften, 42097 Wuppertal, Germany
}

\section{Abstract}

To study the acoustic properties of the Antarctic ice the South Pole Acoustic Test Setup (SPATS) was installed in the upper part of drill holes for the IceCube neutrino observatory. An important parameter for the design of a future acoustic neutrino telescope is the acoustic background noise in the ice and its spatial and temporal variations. We study the absolute noise level depth profile from SPATS data and discuss systematic uncertainties. The measured noise is very stable over one year of data taking, and we estimate the absolute noise level to be $<10 \mathrm{mPa}$ in the frequency range from $10 \mathrm{kHz}$ to $50 \mathrm{kHz}$ at depths below $200 \mathrm{~m}$. This noise level is of the same order of magnitude as observed by ocean based acoustic neutrino detection projects in good weather conditions.

Key words: Acoustic neutrino detection, Acoustic ice properties, SPATS

PACS: 43.50.+y, 43.58.+z, 93.30.Ca

\section{Introduction}

Hadronic processes at highest energies which are anticipated to take place in cosmic sources like Active Galactic Nuclei or Gamma-ray bursts can be studied by observation of the ultra-high energy (UHE) neutrinos which are produced in these interactions (see [1] for a recent review). Further on, there should be a guaranteed flux of UHE neutrinos from the interaction of high energy cosmic ray protons with the cosmic microwave background radiation, the GZK neutrinos 2]. Since the theoretically predicted neutrino fluxes are very low, large detector masses are needed to detect UHE neutrinos with significant statistics. The required sensor density of neutrino telescopes in natural media is determined by the signal attenuation length. While the attenuation length for optical Čerenkov light in polar ice is of the order of $100 \mathrm{~m}$, much larger attenuation lengths, $>1 \mathrm{~km}$, are predicted for radio and acoustic waves from available data 34]. Both types of signals are emitted from the electromagnetic and hadronic cascades generated in an UHE neutrino interaction 56. The Antarctic ice sheet offers the unique possibility to combine these three detection techniques - optical, radio, and acoustic - into a hybrid detector. This will largely increase event tagging and back-

Email address: karg@physik.uni-wuppertal.de (Timo Karg). URL: http://icecube.wisc.edu/ (IceCube collaboration). ground rejection capabilities for the rare UHE neutrino events.

At the site of the IceCube neutrino observatory two test systems have been deployed to study the properties of the South Pole ice: the Askaryan Underice Radio Array (AURA) [7] to measure the radio properties, and the South Pole Acoustic Test System (SPATS) 8] to study the acoustic properties. The quantities of interest are the attenuation length, the sound speed profile which determines the refraction of the acoustic signal, the origin and temporal and spatial distribution of transient backgrounds, and the background noise level which is discussed in this work.

SPATS consists of four instrumented strings that are installed in the upper part of IceCube holes. Each string comprises seven acoustic sensors and seven transmitters. Three of the strings (Strings A, B, and C) were deployed in the austral summer 2006/07 and cover the depth range from $80 \mathrm{~m}$ to $400 \mathrm{~m}$. The array was extended in December 2007 by a fourth string (String D) with second generation sensors and transmitters between $190 \mathrm{~m}$ and $500 \mathrm{~m}$ depth. Horizontal baselines from $125 \mathrm{~m}$ to $543 \mathrm{~m}$ are covered by the setup.

The absolute level and spectral shape of the continuous noise determine the threshold at which neutrino induced signals can be extracted from the background and thus set the lower energy threshold for a given detector configuration. The continuous noise is monitored in SPATS through 
an untriggered read out of all sensor channels for $0.5 \mathrm{~s}$ at $200 \mathrm{kHz}$ sampling rate once every hour.

Transient acoustic noise sources which can be misidentified as possible neutrino candidates in acoustic neutrino telescopes are studied in a triggered run mode. A threshold trigger in any channel can be used to read out up to three channels on the same string. This allows the characterization of transient signals as function of threshold and frequency content (waveform shape).

\section{Properties of the measured noise}

SPATS is operating successfully and has been taking data continuously since its installation and commissioning in January 2007. The untriggered noise recordings show a Gaussian distribution of the ADC counts in each of the 73 channels continuously operating during the considered time interval.

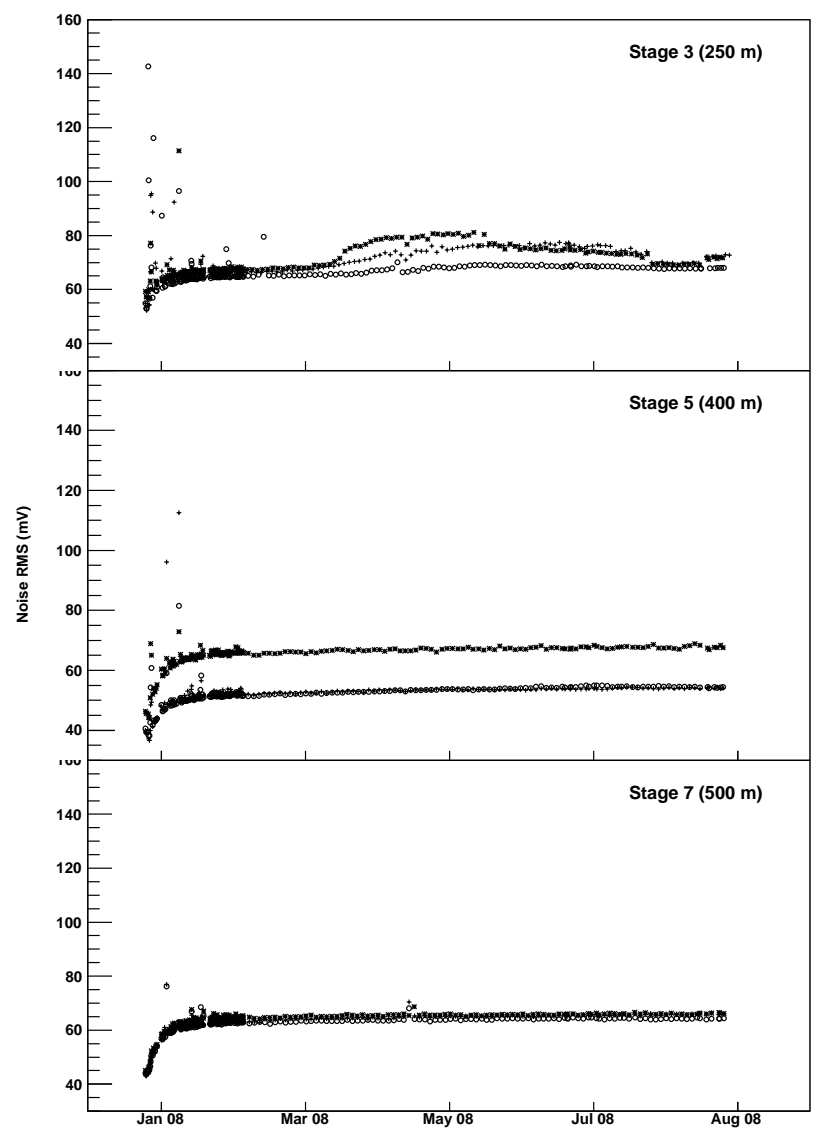

Fig. 1. Evolution of the noise RMS for three exemplary sensors from String D starting from deployment in December 2007. The three different symbols in each panel represent the three sensor channels available at each depth. The excess points in the noise level in January and February are correlated in time with IceCube drilling activity.

Figure 1 shows the RMS of the Gaussian distribution as a function of time for the whole data taking period of String D, starting 26 December 2007. It can be seen, that the noise level stabilizes two to three weeks after deploy- ment in all channels, with the exception of two channels on Stage 3 which is believed to be an instrumental problem. The continuous increase of the measured noise level at the beginning is assumed to be due to an increasing sensitivity at low temperatures and an improved coupling to the bulk ice after freeze-in. The typical deviation from the mean noise level is $\frac{\sigma_{\mathrm{RMS}}}{\langle\mathrm{RMS}\rangle}<10^{-2}$.

The excess points in the noise data before 25 January 2008 are correlated in time to the drilling of IceCube holes. The noise level in each channel rises for few minutes when the drill head passes the sensor depth level on its way down and up again. The drill noise was observed up to the furthest hole drilled in the 2007/08 season which had a horizontal distance of $660 \mathrm{~m}$.

\section{Environmental conditions and absolute calibration}

The determination of the absolute noise level from the measured voltage requires calibration of the sensors. The SPATS sensors have been calibrated in liquid water at $0^{\circ} \mathrm{C}$ in the frequency range from $10 \mathrm{kHz}$ to $80 \mathrm{kHz}$ [9] prior to deployment. However, it is unknown how the sensitivity changes when the sensor is deployed in Antarctic ice, i.e. under the combined influence of low temperatures $\left(-50^{\circ} \mathrm{C}\right)$ and high static pressure. External pressure is produced by the water column inside the drill hole during deployment and increases during freeze in since the hole freezes from the top, creating a water filled cavity. After the hole is frozen completely relaxation of the freshly frozen "hole ice" to the surrounding bulk ice occurs.

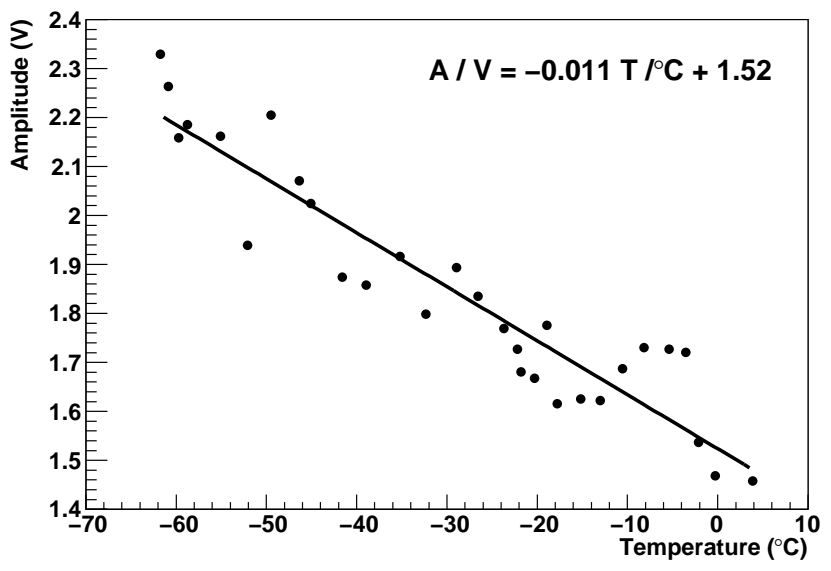

Fig. 2. Peak to peak amplitude of a transmitter signal measured in air with a SPATS sensor as a function of ambient temperature 10] (dots), and linear fit to the data. The measured amplitude is used as an estimator for the sensitivity of the sensor.

Lab measurements have shown that the sensitivity of the SPATS sensor in air at atmospheric pressure increases by a factor of 1.4 when cooled down from $0{ }^{\circ} \mathrm{C}$ to $-50{ }^{\circ} \mathrm{C}$ (cf. Figure 2). In this measurement the recorded peak to peak amplitude of a pulse emitted from a piezoelectric transmitter 
driven by a fixed input voltage and at the same ambient temperature as the receiver is used as an estimator for the sensitivity of the sensor. We use the value of 1.4 to estimate the unknown change of the sensor sensitivity deep in Antarctic ice. A measurement of the sensitivity at room temperature as a function of static pressure was performed in a pressure vessel. Preliminary results indicate a change in sensitivity of $<20 \%$ in the pressure range from 1 bar to 100 bar.

An additional source of systematic uncertainty under study is the influence of the "hole ice": the ice that is freshly refrozen in the IceCube holes after drilling. This ice has a lattice structure different from the bulk ice and so the propagation and attenuation of acoustic signals in this ice column is difficult to describe and can influence the observed noise level. It is expected that under the ambient pressure a reordering takes place in the ice and the "hole ice" becomes compact bulk ice. The time scale for this process however is not known yet.

\section{Estimation of the absolute noise level}

The properties of the continuous noise can be described best in terms of the power spectral density (PSD). We chose the following definition of the PSD of a digitized signal of $N$ samples $X_{k}(k=0 \ldots N-1)$ recorded with sampling frequency $f_{s}$ :

$\operatorname{PSD}_{j}=\frac{2\left|\tilde{X}_{j}\right|^{2}}{f_{s}(N-1)}$, with $\tilde{X}_{j}=\sum_{k=0}^{N-1} X_{k} e^{-2 \pi i \frac{j k}{N}}$.

The coefficient $\mathrm{PSD}_{j}$ corresponds to a frequency of $f_{j}=$ $\frac{f_{s}}{N} \cdot j,(j=0 \ldots N / 2)$. With this definition the integral over the PSD is equal to the RMS of the signal in the time domain, bandwidth limited to the chosen frequency range.

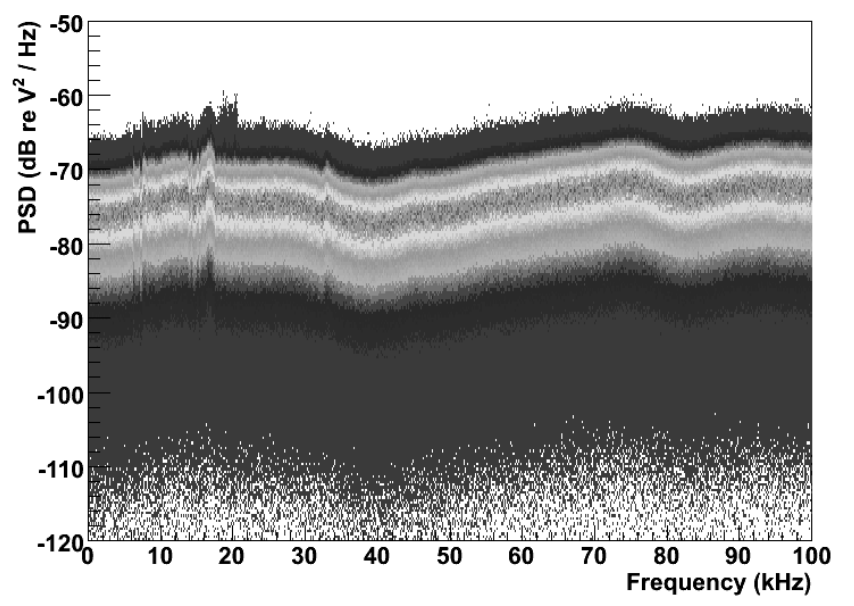

Fig. 3. Long-term distribution of power spectral densities for string $\mathrm{C}$, sensor 7 , channel $2(400 \mathrm{~m})$.

Figure 3 shows the distribution of PSD values in each frequency bin for sensor C7(2) (String C, sensor 7, chan- nel 2) which is at a depth of $400 \mathrm{~m}$. The graph includes all available data from 1 January 2008 to 7 May 2008. A comparison of all sensors shows that the spectral shape of the noise is consistent for all three channels within one sensor housing but differs strongly between different sensors. This is an indication that the spectral shape is determined mostly by the steel housing of the sensor, and the three channels in one sensor are strongly coupled by the housing and a central preload screw. This effect will be studied further by using the second generation sensors that were installed on the fourth SPATS string. These include SPATS type steel sensors with modified coupling of the piezo element to the housing and HADES sensors [1] comprised of a piezo element and pre-amplifier enclosed in resin.

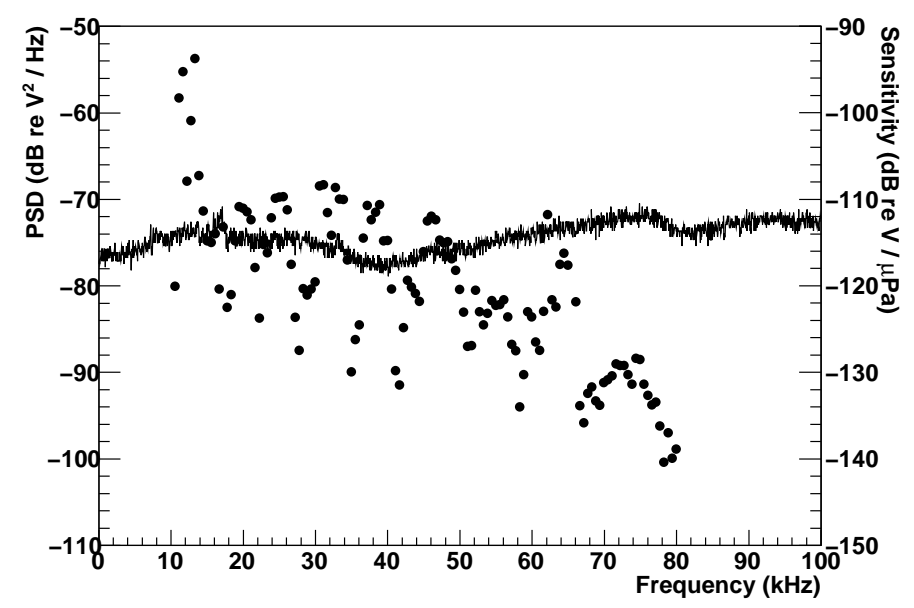

Fig. 4. Mode (solid line) calculated per frequency bin of the distribution shown in Figure 3 Superimposed is the sensitivity of the sensor measured in water scaled by a factor of 1.4 (dots, cf. text).

To reduce the data further the distribution of PSD values in each frequency bin is evaluated. In Figure 4 the mode for each frequency bin is shown, which is a measure for the most common noise level.

Also shown in Figure 4 is the sensitivity measured in water scaled by a factor of 1.4 as discussed in Section 3 . Since the sensitivity decreases strongly at high frequencies, only the frequency range from $10 \mathrm{kHz}$ to $50 \mathrm{kHz}$ is considered for further analysis.

After the scaled sensitivity is applied to the PSD mode, the resulting pressure PSD is integrated over the relevant frequency range from $10 \mathrm{kHz}$ to $50 \mathrm{kHz}$ to determine the absolute noise level RMS. Figure 5 shows the mean noise level as function of sensor depth. The error bars represent the standard deviation of the values measured in the up to 12 sensor channels available at each depth.

It can be seen that both the noise level and fluctuations are high in the upper $200 \mathrm{~m}$ of the SPATS setup. In this region, the firn, a transition takes place from a snow/air mixture to compact bulk ice. Below the firn, the noise conditions become more stable and an average noise level below $10 \mathrm{mPa}$ in the frequency range relevant for acoustic neutrino detection $(10 \mathrm{kHz}$ to $50 \mathrm{kHz})$ is derived. 


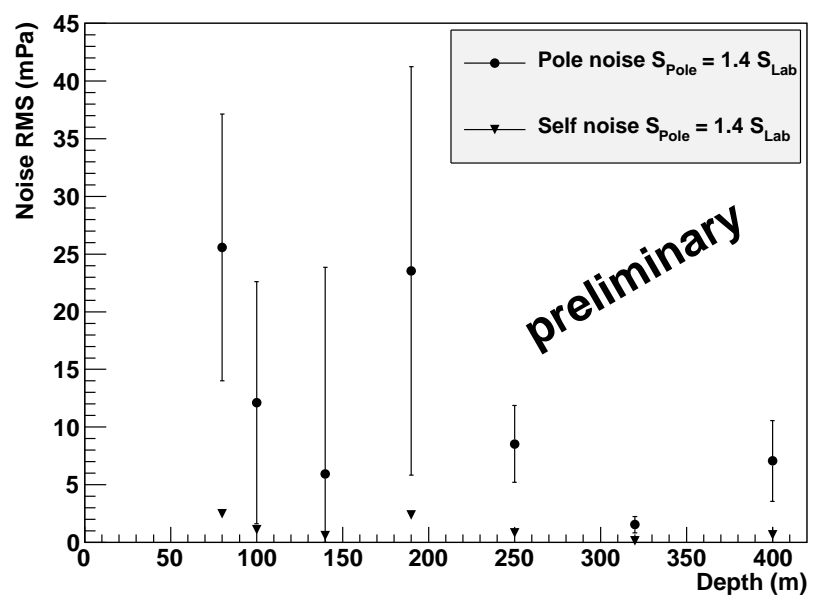

Fig. 5. Noise RMS integrated from $10 \mathrm{kHz}$ to $50 \mathrm{kHz}$ as function of sensor depth. The error bars only describe sensor to sensor variations.

\section{Conclusions}

The South Pole Acoustic Test Setup is successfully recording valuable data to study the acoustic properties of deep South Pole ice with respect to the feasibility of acoustic UHE neutrino detection for one and a half years. We have shown that the level of background noise, which is a critical parameter for the construction of an acoustic neutrino telescope, is stable up to the time scale of one year. A first estimation of the absolute noise level at depths larger than $200 \mathrm{~m}$ indicates values below $10 \mathrm{mPa}$ integrated over the relevant frequency range for neutrino detection from $10 \mathrm{kHz}$ to $50 \mathrm{kHz}$. This value is of the same order of magnitude as what is observed in marine studies at good weather conditions 1213. However, this result is subject to systematic uncertainties which are under study. These include the change of sensor sensitivity under the combined exposure to low temperatures and high ambient pressure and the influence of the freshly frozen "hole ice" the sensors are emerged in.

Acknowledgements

We gratefully acknowledge the hospitality of the National Science Foundation Amundsen-Scott South Pole Station.

This work was supported by the German Ministry for Education and Research.

\section{References}

[1] J.K. Becker, Phys. Rep. 458 (2008) 173, arXiv:0710.1557 [astro-ph].

[2] V.S. Beresinsky and G.T. Zatsepin, Phys. Lett. B 28 (1969) 423.

[3] S. Barwick et al., J. Glaciol. 51 (2005) 231.

[4] P.B. Price, J. Geophys. Res. 111 (2006) B02201.
[5] G.A. Askaryan, Sov. Phys. JETP 14 (1962) 441.

[6] G.A. Askaryan, At. Energ. 3 (1957) 152.

[7] H. Landsman et al., 30th International Cosmic Ray Conference, Mérida, México, July 2007, arXiv:0711.0353 [astro-ph].

[8] S. Böser et al., 30th International Cosmic Ray Conference, Mérida, México, July 2007, arXiv:0807.4676 [astro-ph].

[9] J.-H. Fischer, Diploma Thesis, Humboldt-Universität zu Berlin (2006).

[10] M. Bothe, Diploma Thesis, in preparation, HumboldtUniversität zu Berlin (2008).

[11] B. Semburg, these Proceedings (2008).

[12] N. Kurahashi and G. Gratta, submitted to Phys. Rev. D (2008) arXiv:0712.1833 [physics.ao-ph].

[13] S. Aiello et al., submitted to Deep Sea Research I (2008) arXiv:0804.2913 [astro-ph]. 